\title{
Gender differences in sclerostin and clinical characteristics in type 1 diabetes mellitus
}

\author{
Antonino Catalano, Basilio Pintaudi ${ }^{1}$, Nancy Morabito, Giacoma Di Vieste, \\ Loretta Giunta, Maria Lucia Bruno, Domenico Cucinotta, Antonino Lasco and \\ Antonino Di Benedetto
}

Department of Clinical and Experimental Medicine, University Hospital of Messina, A.O.U. Policlinico 'G.Martino', Via C. Valeria, 98125 Messina, Italy and ${ }^{1}$ Department of Clinical Pharmacology and Epidemiology, Fondazione Mario Negri Sud, Santa Maria Imbaro (CH), Italy
Correspondence should be addressed to A Catalano

Email

catalanoantonino@libero.it

\begin{abstract}
Background: Sclerostin is an osteocyte-derived inhibitor of the Wnt/ $\beta$-catenin signaling pathway, which acts as a negative regulator of bone formation. Published data on sclerostin levels in type 1 diabetes mellitus (T1DM) are few.

Objective: To evaluate gender differences in sclerostin serum levels and the association among sclerostin, bone mass, bone metabolism, and the main clinical characteristics of subjects with T1DM.

Design and methods: A total of 69 patients with T1DM (mean age, 33.7 $\pm 8.1 ; 49 \%$ males) were enrolled in this cross-sectional study in a clinical research center. Bone mineral density was measured by phalangeal quantitative ultrasound (QUS); bone turnover markers (urinary pyridinoline, deoxypyridinoline (D-PYR), and urine hydroxyproline (OH-PRO) to evaluate bone resorption; serum bone alkaline phosphatase and BGP to evaluate bone formation) and sclerostin were assessed.

Results: D-PYR and sclerostin were significantly higher in women when compared with men $(P=0.04)$. A disease duration $>15$ years was associated with higher sclerostin levels $(P=0.03)$. Bone turnover markers and QUS parameters were not correlated with sclerostin. A significant negative correlation was observed among QUS parameters, BMI, and OH-PRO. Sclerostin serum levels were correlated with homocysteine $(r=-0.34, P=0.005)$ and vitamin $\mathrm{B} 12(r=-0.31, P=0.02)$. Generalized linear model showed that macroangiopathy was the only predictor of sclerostin serum levels $(\beta=-11.8$, $95 \% \mathrm{Cl}$ from -21.9 to $-1.7 ; P=0.02$ ).

Conclusions: Our data demonstrate that women with T1DM exhibit higher sclerostin levels than men and that circulating sclerostin is not associated with bone turnover markers and phalangeal QUS measurements. Macroangiopathy was associated with sclerostin levels.
\end{abstract}

\section{Introduction}

Several studies have demonstrated a strong association between diabetes mellitus (DM) and bone impairment, which lead to an increasing fracture risk in subjects with type 2 DM (T2DM), but mainly in subjects with T1DM (1). A reduced bone formation as a consequence of reduced osteoblast activity has been identified as the mean pathogenetic effect or for low bone mineral density (BMD) in subjects with T1DM $(1,2,3,4,5,6,7,8,9,10$, $11,12,13,14,15,16,17,18,19,20,20,21,22,23,24,25$, $26,27,28,29,30,31,32,33,34,35,36,37,38,39$ ).
Recently, the Wnt signaling pathway has been recognized as a major trigger of osteoblastic proliferation and differentiation, leading to bone formation (8). Moreover, WNT proteins are known to play a pivotal role in development and homeostasis processes by regulating cell differentiation, proliferation, and apoptosis in different tissues comprehensive of bone $(4,5,6)$. Emerging evidences are showing that the Wnt signaling pathway is involved in cardiovascular pathophysiology $(7,8)$. Vascular smooth muscle cell (VSMC) proliferation causes 
intimal thickening in atherosclerosis, and $\mathrm{Wnt} / \beta$-catenin signaling is a regulator of VSMC behavior (9). WNT proteins have been detected in murine and human atherosclerotic lesions suggesting a role for Wnt signaling in atherosclerosis (10).

The Wnt signaling pathway is modulated by a number of factors that include, among others, sclerostin, which has been identified as a main regulator of bone formation. By inhibiting the Wnt signaling pathway, sclerostin reduces osteoblast proliferation and function, thereby decreasing bone formation. Encoded by the gene SOST, sclerostin is expressed almost exclusively in the osteocytes that represent $95 \%$ of all bone cells. The role of sclerostin in bone homeostasis is underlined by two rare skeletal human diseases, i.e. van Buchem disease and sclerosteosis, characterized by high bone mass and impaired sclerostin production (11). However, experimental and clinical evidences demonstrated the expression of sclerostin and WNT molecules also in calcifying vascular tissues $(12,13)$. Sclerostin has been localized in the subendothelial layer of aortic intima in human aortic samples of atherosclerosis (14), giving new insights into the association between atherosclerosis and osteoporosis (15), and supporting the hypothesis that osteoporosis and cardiovascular diseases could be, at least in part, the pleiotropic consequences of impaired Wnt signaling.

Examining a cohort of patients with T1DM and T2DM, Gennari et al. (16) reported that subjects with T2DM but not those with T1DM showed higher sclerostin serum levels when compared with healthy matched controls. Differently, Neumann et al. (17) have recently reported that subjects with T1DM exhibited higher sclerostin concentrations in comparison with controls.

To our knowledge, a gender difference in sclerostin levels and the possible association between circulating sclerostin concentrations and patient clinical characteristics, including diabetes-related complications, have not yet been reported in subjects with T1DM.

The aims of this study were to evaluate the gender differences in bone mass and metabolism and to analyze the relationship between sclerostin and the main clinical characteristics in a wide cohort of patients with T1DM.

\section{Methods}

\section{Study design and participants}

Our cross-sectional study included a group of Caucasian subjects with T1DM, consecutively referred to the Diabetic
Outpatients Clinic of the Department of Clinical and Experimental Medicine, University Hospital of Messina (Messina, Italy) from January 2010 to May 2011. Exclusion criteria were as follows: age $<18$ years, pregnancy, current or previous use of oral contraceptives, corticosteroids, or other medications known to affect bone and mineral metabolism (including calcium and vitamin D; with the only exception of levothyroxine for the treatment of hypothyroidism), chronic renal failure, celiac disease, or other diseases affecting bone health. Owing to the known increase in sclerostin serum levels caused by estrogen deprivation in postmenopausal women, four postmenopausal women were excluded from the analysis in this study. All the recruited subjects did not show any clinical signs of hypogonadism. The study was conducted with the approval of the Local Ethics Committee and conformed to the Declaration of Helsinki. Written informed consent was obtained from all subjects before participating in this study.

\section{Clinical evaluation}

Height and weight were measured at baseline according to standard procedures and the BMI was calculated as weight divided by the square of height in meters. For each patient, information about the presence of diabetic complications recorded in clinical charts was evaluated. In particular, macrovascular disease was defined as a history of a cardiovascular event and/or ischemic electrocardiogram abnormalities at rest or in a stress test, or the presence of plaques detected by ultrasonographic examination of the carotid arteries or the peripheral arterial vessels or as the presence of an intima media of thickness $>1.5 \mathrm{~mm}$ (18); sensory-motor neuropathy was diagnosed by the vibration perception test, the monofilament pressure sensation test, or electromyography; nephropathy was defined as an increased urinary albumin excretion (albuminuria) diagnosed if urinary albumin concentration was $>30 \mathrm{mg} / \mathrm{l}$, or if urinary albumin excretion rate was $>20 \mu \mathrm{g} / \mathrm{min}$, or if urinary albumin-to-creatinine ratio was $>2.5 \mathrm{mg} / \mathrm{mmol}$ in men and $3.5 \mathrm{mg} / \mathrm{mmol}$ in women; and retinopathy was detected by high-quality fundus photographs (19). All subjects were free from diseases or comorbidities impairing normal daily activity. Further information regarding duration of disease, total daily insulin requirements, and insulin therapeutic schemes (continuous subcutaneous insulin infusion (CSII) system or multiple daily injections) was collected. Participants reported alcohol use, smoking status, and level of physical activity in a specific health questionnaire. 


\section{Biochemical measurements}

Biochemical evaluation was conducted on serum and 2-h morning urine samples obtained after an overnight fast. Serum and urinary calcium, phosphorus, serum and urinary creatinine, alkaline phosphatase (ALP), bone ALP (B-ALP), as a marker of bone formation, and $\mathrm{HbA1c}$ were measured using standard laboratory techniques; PTH, folic acid, vitamin B12, 25-hydroxyvitamin D and urine hydroxyproline (OH-PRO), pyridinoline (PYR), and deoxypyridinoline (D-PYR), as markers of bone resorption, were determined by HPLC; and osteocalcin (BGP), a marker of bone formation, and homocysteine levels were measured by immunoenzymatic assay. Serum sclerostin was measured using a commercially available ELISA Kit (Biomedica, Vienna, Austria) according to the manufacturer's instructions with intra- and inter-assay coefficient of variation $(\mathrm{CV})$ values of 5 and $6 \%$ respectively.

\section{Bone mass measurements}

To assess bone mass, we performed quantitative ultrasound (QUS) measurements at the proximal phalangeal metaphysis of the last four fingers of the nondominant hand using a DBM Sonic Bone Profiler (Igea, Carpi, Italy), as described previously (20). Briefly, two probes mounted on a precision caliper, one acting as generator of the signal and the other one as receiver, were positioned on the lateral and medial surfaces of each finger. Standard ultrasound gel was used to couple probes to the skin. A Plexiglass phantom served to calibrate the device as recommended by the manufacturer. The following parameters were measured: amplitude-dependent speed of sound (AD-SoS), bone transmission time (BTT), fastwave amplitude (FWA), signal dynamic (SDy), and ultrasound bone profile index (UBPI) calculated automatically $(\mathrm{UBPI}=-(0.0018 \times \mathrm{SDy}-0.0560 \times \mathrm{FWA} \quad 0.0560-1.1467$ $\times$ BTT +3.0300$)$ ). In accordance with the reference data from the manufacturer, $T$ - and $Z$-score values were also evaluated. The same trained operator, who was blinded to clinical parameters, performed the QUS measurements. The $\mathrm{CV}$ value, determined by repeated measurements in a subgroup of 25 subjects, was of $0.9 \%$.

\section{Statistical analysis}

Data were expressed as means \pm s.D. for continuous variables and percentages for categorical variables. Characteristics of the study population were categorized by gender. The Kolmogorov-Smirnov test was used to test the normality of distribution of continuous variables. For continuous variables, mean values between two groups were compared by unpaired Student's $t$-test for normally distributed variables and the Mann-Whitney $U$ test for skewed variables. The $\chi^{2}$-test was used to compare categorical variables between groups. Pearson's correlation coefficient was employed to test correlations among sclerostin, bone turnover markers, and QUS parameters. To determine the independent variables correlated with sclerostin (dependent variable), a generalized linear model was performed. The following variables were included in the model: gender, age, BMI, duration of disease, HbA1c, macrovascular disease, retinopathy, and neuropathy. A $P$ value of $<0.05$ was considered as statistically significant. Statistical analysis was performed using the Statistical Package for Social Science Software (SPSS Statistics, 17.0).

\section{Results}

Overall, 69 subjects with T1DM were recruited and their clinical data are shown in Table 1. All participants were considered together and divided into two groups according to gender. When considering T1DM subjects by gender, there were no statistical differences in age, BMI, duration of disease, smoking, HbA1c, and prevalence of retinopathy and neuropathy. A statistical difference between groups was shown regarding insulin treatment schemes, women using more CSII devices, and in macroangiopathy, men in whom this condition occurs more frequently.

Food energy, estimated mean calcium intake, alcohol consumption, physical activity, and insulin doses were not significantly different in men in comparison with women (data not shown).

QUS parameters were not significantly different between men and women (Table 2). No significant difference by gender was observed regarding bone formation markers (B-ALP and BGP); differently, urinary level of D-PYR, a marker of bone resorption, was significantly higher in women revealing a slight increased turnover rate in comparison with men.

Sclerostin serum levels were higher in women, even after adjustment for BMI and age ( $P=0.04$; Fig. 1). However, sclerostin was not significantly different in all T1DM subjects in comparison with a group of ten agematched healthy controls $(13.33 \pm 11.14$ vs $14.21 \pm 13.15$ respectively; $P>0.05$; Table 1$)$. When considering the duration of disease, subjects of both genders showed higher serum sclerostin levels in case of a disease duration greater than the median value of 15 years, when compared 
Table 1 Clinical characteristics of the study population according to gender.

\begin{tabular}{|c|c|c|c|c|c|c|c|c|}
\hline & \multicolumn{4}{|c|}{ T1DM subjects } & \multicolumn{4}{|c|}{ Controls } \\
\hline & Overall $(n=69)$ & Males $(n=34)$ & Females $(n=35)$ & $P$ & Overall $(n=10)$ & Males $(n=5)$ & Females $(n=5)$ & $P$ \\
\hline Age (years) & $33.7 \pm 8.1$ & $32.8 \pm 9.3$ & $34.5 \pm 6.9$ & 0.39 & $32.8 \pm 8.0$ & $32.4 \pm 8.7$ & $32.2 \pm 8.3$ & 0.88 \\
\hline BMI $\left(\mathrm{kg} / \mathrm{m}^{2}\right)$ & $24.5 \pm 3.4$ & $24.8 \pm 3.6$ & $24.3 \pm 3.2$ & 0.52 & $24.1 \pm 2.2$ & $24.2 \pm 2.1$ & $24.0 \pm 2.1$ & 0.94 \\
\hline Smokers (\%) & 36.2 & 29.4 & 42.9 & 0.18 & 30 & 40 & 20 & 0.80 \\
\hline Duration of disease (years) & $15.4 \pm 8.8$ & $14.9 \pm 9.3$ & $15.9 \pm 8.4$ & 0.64 & & & & \\
\hline Patients with CSII (\%) & 30.4 & 17.6 & 42.9 & 0.02 & & & & \\
\hline $\mathrm{HbA1c}$ (proportion of total $\mathrm{Hb}$ ) & $0.08 \pm 0.01$ & $0.08 \pm 0.02$ & $0.08 \pm 0.1$ & 0.90 & & & & \\
\hline \multicolumn{9}{|l|}{ Diabetic complications (\%) } \\
\hline Macrovascular disease & 14.5 & 23.5 & 5.7 & 0.04 & & & & \\
\hline Nephropathy & 7.2 & 5.9 & 8.6 & 0.67 & & & & \\
\hline Retinopathy & 37.7 & 44.1 & 31.4 & 0.20 & & & & \\
\hline Neuropathy & 13.0 & 5.9 & 20.0 & 0.08 & & & & \\
\hline
\end{tabular}

with a lower duration of disease $(16.5 \pm 11.3$ vs $10.3 \pm 10.3$ respectively; $P=0.03$ ), even after adjusting for age and BMI; this difference disappeared when adjusting even for impairment of renal function $(P=0.56)$.

Sclerostin levels differed, but not significantly, in patients with macroangiopathy $(7.9 \pm 7.0$ vs $13.8 \pm 11.4$ for macroangiopathy yes vs macroangiopathy no, $P=0.18$; Fig. 2), retinopathy $(15.6 \pm 12.9$ vs $11.7 \pm 10.0$, for retinopathy yes vs retinopathy no, $P=0.34$ ), and neuropathy $(21.3 \pm 16.3$ vs $11.8 \pm 9.6$ for neuropathy yes vs neuropathy no, $P=0.12)$. Men with T1DM showed significantly higher homocysteine levels albeit no differences in folate and vitamin B12 levels were detected in comparison with women (Table 2). A significant negative correlation was observed between the BMI and UBPI $(r=-0.32, P=0.008)$, AD-SoS $\quad(r=-0.52, \quad P<0.0001), \quad T$-score $\quad(r=-0.52$, $P<0.0001)$ and $Z$-score $\quad(r=-0.44, \quad P<0.0001)$.
QUS parameters were also correlated with OH-PRO $(r=-0.26, \quad P=0.03 ; \quad r=-0.32, \quad P=0.009 ; \quad r=-0.27$, $P=0.003$; and $r=-0.40, P=0.001$ for AD-SoS, UBPI, $T$-score, and $Z$-score respectively) and PYR $(r=-0.34$, $P=0.006$ for AD-SoS, UBPI, and $Z$-score; $r=-0.38$, $P=0.002$ for $T$-score). Sclerostin serum levels were correlated with vitamin $\mathrm{B} 12(r=-0.31, P=0.02)$ and homocysteine ( $r=-0.34, P=0.005$ ) levels but not with bone turnover markers or QUS measurements. A generalized linear model showed that macroangiopathy was the only predictor of sclerostin serum levels in the whole study population $(\beta=-11.8,95 \%$ CI from -21.9 to $-1.7 ; P=0.02)$.

\section{Discussion}

Our study investigated bone mass and metabolism in subjects affected by T1DM and explored the relationship

Table 2 Laboratory data and phalangeal quantitative ultrasound measurements according to gender.

Calcium $(\mathrm{mmol} / \mathrm{l})$

Phosphorus (mmol/l)

Serum creatinine $(\mathrm{mmol} / \mathrm{l})$

25-Hydroxyvitamin D (nmol/l)

PTH (ng/l)

Osteocalcin $(\mu \mathrm{g} / \mathrm{l})$

Bone alkaline phosphatase ( $\mu \mathrm{kat} / \mathrm{l})$

Hydroxyproline (pmol/ $\mu \mathrm{mol}$ urinary creatinine)

Pyridinoline ( $\mathrm{pmol} / \mu \mathrm{mol}$ urinary creatinine)

Deoxypyridinoline ( $\mathrm{pmol} / \mu \mathrm{mol}$ urinary creatinine)

Urinary calcium/urinary creatinine ratio

Sclerostin (pmol/l)

Homocysteine $(\mu \mathrm{mol} / \mathrm{l})$

Folic acid (nmol/l)

Vitamin B12 (pmol/l)

AD-SoS $(\mathrm{m} / \mathrm{s})$

UBPI (U)

T-score (s.D.)

Z-score (S.D.)

\begin{tabular}{c} 
Overall $(n=69)$ \\
\hline $2.23 \pm 0.1$ \\
$1.23 \pm 0.19$ \\
$61.88 \pm 17.68$ \\
$66.14 \pm 26.71$ \\
$16.6 \pm 5.8$ \\
$6.2 \pm 5.9$ \\
$0.63 \pm 0.16$ \\
$30.1 \pm 15.8$ \\
$59.0 \pm 32.1$ \\
$15.4 \pm 7.7$ \\
$0.09 \pm 0.08$ \\
$13.1 \pm 11.1$ \\
$11.2 \pm 5.1$ \\
$14.5 \pm 17.45$ \\
$468.72 \pm 331.49$ \\
$2002.3 \pm 102$ \\
$0.54 \pm 0.19$ \\
$-1.73 \pm 1.45$ \\
$-1.71 \pm 1.6$ \\
\hline
\end{tabular}

\begin{tabular}{c}
\hline Females $(n=35)$ \\
\hline $2.2 \pm 0.08$ \\
$1.23 \pm 0.19$ \\
$53.04 \pm 8.84$ \\
$71.39 \pm 29.95$ \\
$16.6 \pm 5.4$ \\
$6.0 \pm 6.9$ \\
$0.67 \pm 0.15$ \\
$30.9 \pm 12.2$ \\
$65.7 \pm 33.8$ \\
$17.7 \pm 9.1$ \\
$0.08 \pm 0.06$ \\
$15.6 \pm 12.5$ \\
$8.8 \pm 1.6$ \\
$13.82 \pm 6.12$ \\
$446.81 \pm 204.96$ \\
$1994.6 \pm 89.5$ \\
$0.53 \pm 0.17$ \\
$-1.85 \pm 1.28$ \\
$-1.75 \pm 1.26$ \\
\hline
\end{tabular}

\begin{tabular}{c}
\hline \multicolumn{1}{c}{} \\
\hline 0.006 \\
0.78 \\
$<0.0001$ \\
0.11 \\
0.94 \\
0.71 \\
0.08 \\
0.68 \\
0.08 \\
0.01 \\
0.35 \\
0.04 \\
$<0.0001$ \\
0.72 \\
0.60 \\
0.55 \\
0.51 \\
0.54 \\
0.86
\end{tabular}




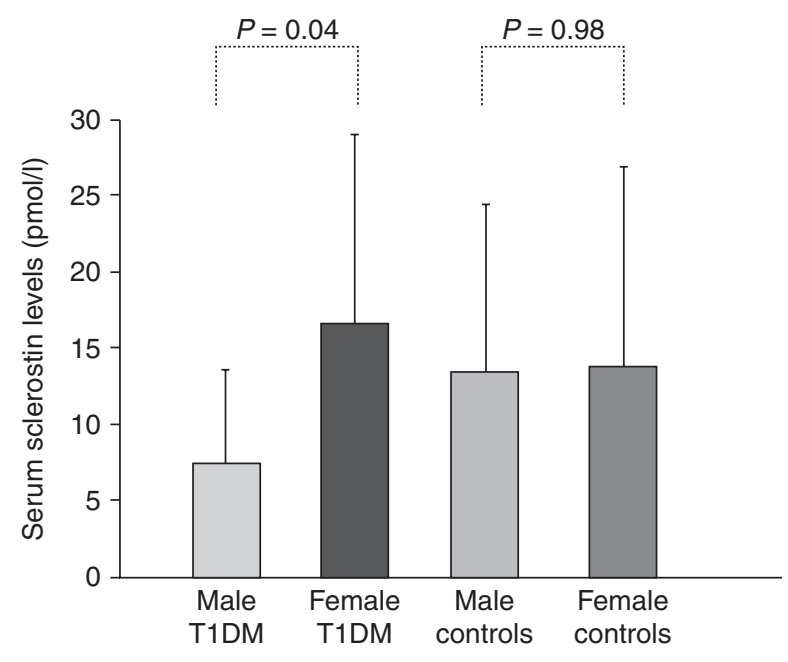

\section{Figure 1}

Serum sclerostin levels according to gender in T1DM and healthy control subjects. Data are expressed as mean \pm s.D.

between sclerostin and the main clinical features of patients with T1DM. Sclerostin is an osteocyte-derived glycoprotein, which plays a key role in the regulation of the canonical Wnt signaling pathway. Sclerostin is critical for osteoblast proliferation and activity, not only leading to inhibition of bone formation, but also encouraging bone resorption (21). Humans with genetic deficiencies of sclerostin and mice with knockout of the Sost gene have high bone mass and increased bone strength. Sclerostin works by binding to the LDL receptor-related proteins 5 and 6 (LRP5 and LRP6), leading to translocation of $\beta$-catenin into the nucleus and to regulation of targeted gene expression $(4,5,6)$. To date, few data have been published on sclerostin in diabetes and only two studies have reported sclerostin serum levels in subjects with T1DM analyzing their relationship with bone turnover markers and bone mass $(16,17)$. However, these cohorts were heterogenous for a number of participants, age, and duration of disease ranges when compared with our study population. Our first interesting and furthermore innovative result was that circulating sclerostin levels were significantly increased in women when compared with men with T1DM. With few exceptions (22), most of the existing data in the literature $(23,24)$ showed higher sclerostin levels in men to an extent diametrically opposed with our findings; furthermore, they are based on studies involving subjects affected by T2DM (30). Even Gennari et al. (16) showed higher sclerostin levels in men but the overall cohort also included T2DM subjects and healthy controls; for this reason, their results are not generalizable to T1DM. Otherwise, after adjusting for age, no differences in sclerostin serum levels by gender were found in subjects with T1DM in the study by Neumann et al. (17). In our opinion, the fact that the women we studied had higher sclerostin levels was not a coincidence, because i) we performed serum sclerostin measurements using the same method and the same commercially available kit that was also used by Gennari et al. and ii) the women we studied showed a higher bone turnover rate when compared with men, and we can reasonably hypothesize that sclerostin may have contributed to the increased bone resorption. Modder et al. (23) studying sclerostin serum levels in a population-based sample of 362 women and 318 men observed that, at any age, serum sclerostin levels were higher in men than in women. They concluded that reasons for this are unclear, except that circulating sclerostin levels might reflect total-body skeletal mass and the larger skeleton in men simply may produce and release more sclerostin into the circulation. This hypothesis can be realistic after the study conducted by Amrein et al. (24), in which men had significantly higher unadjusted sclerostin levels than women but levels were not significantly different after adjustment for skeletal size. Our data raise the issue of the role of sex steroids in explaining gender differences in sclerostin serum levels: both estrogen and testosterone are critical regulators of bone turnover and have been inversely associated with sclerostin levels $(25,26)$. Disturbances in these

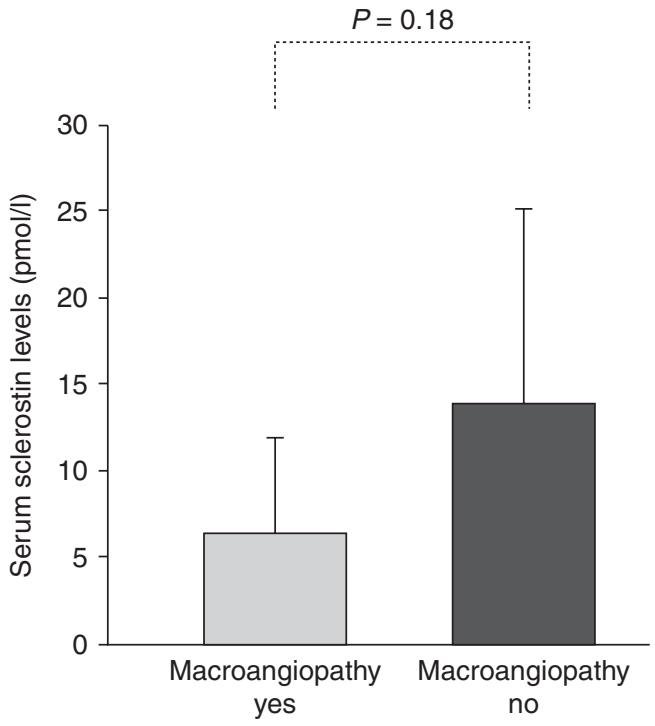

Figure 2

Serum sclerostin levels in T1DM subjects with and without macroangiopathy. Data are expressed as mean \pm s.D. 
hormones were reported previously in T1DM $(27,28,29)$. Although the recruited subjects did not exhibit any clinical sign of hypogonadism or other hormonal disorders, however, we cannot rule out biochemical hormonal abnormalities having not looked at circulating sex hormones. Sclerostin serum levels were significantly increased in women with T1DM who also showed a higher bone turnover rate when compared with men, and we hypothesized that sclerostin may have contributed to the increased bone resorption. Interestingly, we found significantly higher sclerostin serum levels in subjects with long disease duration ( $>15$ years) when compared with subjects without long disease duration ( $<15$ years). As sclerostin level increases with age and it has been suggested to be contributing to age-related bone loss (23), it might be supposed that increased sclerostin levels in long disease duration could play a role in explaining, at least in part, the effects of diabetes duration on bone tissue per se.

In our population, poor QUS values were observed in subjects with T1DM with increased bone resorption, a higher BMI, and low vitamin D levels, but we did not observe an association between bone density detected by phalangeal QUS and sclerostin levels. The latter is consistent with the data reported by Gennari et al. (16) using DXA, although an association between sclerostin serum levels and DXA measurements was also described in T1DM (17). Besides BMD, in previous works, sclerostin was associated with fracture risk, although etherogeneus results were reported $(31,32)$. In particular, sclerostin was related to vertebral fractures in T2DM (33). Data on the association between sclerostin levels and fracture risk in T1DM are still lacking.

The novelty of this research remains the link between sclerostin and macroangiopathy, which has never been reported before in T1DM. Recently, but in a population of subjects with T2DM, Morales-Santana et al. (30) have observed an association between circulating sclerostin and surrogate markers of atherosclerotic disease such as abnormal carotid intima-media thickness (IMT), carotid plaque, and aortic calcification. Sclerostin has been found to be involved in vascular calcification (VC) $(13,14)$, and higher sclerostin levels were detected in postmenopausal women with a higher pulse wave velocity (34). Lower sclerostin serum levels were identified as independent determinants of VC in patients with chronic kidney disease not yet on dialysis, thus it was suggested that circulating sclerostin may be a protective factor for the progression of VC (35). Consistent with this, higher sclerostin levels have been associated with a reduced mortality in hemodialysis patients (36).
As known, cardiovascular disease as a result of macrovascular atherosclerotic progression is the major cause of mortality among patients with diabetes, and atherosclerosis had already been observed in childhood with T1DM. Arterial wall is the second most extensively calcified structure in the human body, and VC is a complex process involving cellular phenotypic switch from VSMCs to mineralizing osteoblast-like cells. These phenotypic changes permit arterial VSMC migration into the intima where these cells are able to express several osteogenic genes such as the osteoblastic transcription factor, Cbfa1/Runx2, bone morphogenetic protein 2 (BMP2), and Msx2 (a promoter of early osteoblast development) in addition to extracellular matrix and matrix-degrading metalloproteinases $(12,13,37)$. The prevalence of coronary atherosclerosis is similar between patients with T1DM and those with T2DM, with a relatively higher proportion of noncalcified plaques observed in patients with T2DM in comparison with those with T1DM (38). As T2DM was reported previously to show higher sclerostin levels than T1DM (16), it is reasonable to speculate that sclerostin could act as an inhibitor of VC in T2DM. At vascular level, $\beta$-catenin activity, under control by $\mathrm{N}$-cadherin and reactive oxygen species (ROS), drives VSMC proliferation and neointima formation, thus sclerostin might attenuate the canonical Wnt signaling pathway stimulated by peroxide via an ROS-sensitive nucleoredoxin-disheveled relay (39). Therefore, it is likely that macroangiopathy in T1DM could be linked to lower sclerostin serum levels. In our study, women exhibited higher sclerostin levels and a lower prevalence of macroangiopathy when compared with men who, on the other hand, showed higher homocysteine levels. Sclerostin was negatively associated with homocysteine in the whole population of T1DM, in contrast to what has recently been observed in T2DM (30). These data may account for an increased cardiovascular risk in men in comparison with women with T1DM. In addition, our results seem to be in line with a recent report showing that, in T2DM postmenopausal women, sclerostin was negatively associated with IMT, and was an independent predictor of IMT in multivariate linear regression analysis (40). Taken together with our findings, these evidences suggest that sclerostin may be involved in the delay of atherosclerosis progression.

In conclusion, our study is the first one that has investigated the relationship between serum sclerostin levels and the main clinical characteristics in subjects with T1DM according to gender, suggesting that macroangiopathy was predictive of circulating sclerostin. We must 
recognize, however, some limitations of this research: the relatively small sample size to account for more specific associations between sclerostin and T1DM-related complications and the lack of DXA measurements of bone mass, although we used a technique that was found to correlate with DXA. Moreover, this being a cross-sectional study no causal inferences can be made; in fact, the cross-sectional design does not allow establishing a cause-effect relationship between sclerostin and macroangiopathy. Future longitudinal studies are required to investigate the role of sclerostin in vascular pathology. Observing changes in sclerostin levels over time and correlating these changes with those observed in metabolic and vascular morphological parameters could provide a contribution far greater than our results. Addressing this issue is critical, as inhibition of sclerostin, via sclerostin-neutralizing antibodies, is an upcoming intriguing anabolic treatment for osteoporosis, but the potential cardiovascular effects should be carefully evaluated even in subjects with T1DM.

\section{Declaration of interest}

The authors declare that there is no conflict of interest that could be perceived as prejudicing the impartiality of the research reported.

\section{Funding}

This research did not receive any specific grant from any funding agency in the public, commercial or not-for-profit sector.

\section{Acknowledgements}

The authors would like to thank Dr Santa Brancatelli for the sclerostin assay.

\section{References}

1 Hofbauer LC, Brueck CC, Singh SK \& Dobnig H. Osteoporosis in patients with diabetes mellitus. Journal of Bone and Mineral Research 200722 1317-1328. (doi:10.1359/jbmr.070510)

2 Rakel A, Sheehy O, Rahme E \& LeLorier J. Osteoporosis among patients with type 1 and type 2 diabetes. Diabetes \& Metabolism 200834 193-205. (doi:10.1016/j.diabet.2007.10.008)

3 Danielson KK, Elliott ME, LeCaire T, Binkley N \& Palta M. Poor glycemic control is associated with low BMD detected in premenopausal women with type 1 diabetes. Osteoporosis International 200920 923-933. (doi:10.1007/s00198-008-0763-3)

4 MacDonald BT, Tamai K \& He X. Wnt/ $\beta$-catenin signaling: components, mechanisms, and diseases. Developmental Cell 200917 9-26. (doi:10.1016/j.devcel.2009.06.016)

5 Nelson WJ \& Nusse R. Convergence of Wnt, $\beta$-catenin, and cadherin pathways. Science 2004303 1483-1487. (doi:10.1126/science.1094291)

6 Moon RT, Kohn AD, De Ferrari GV \& Kaykas A. Wnt and $\beta$-catenin signaling: diseases and therapies. Nature Reviews. Genetics 20045 691-701. (doi:10.1038/nrg1427)
7 Mill C, Tsaousi A, Woodward E, Johnson J \& George S. Increased expression of Wnt5A in unstable atherosclerotic plaques is associated with increased MMP expression and may contribute to instability. Atherosclerosis 2010213 E12. (doi:10.1016/j.atherosclerosis.2010. 08.022)

8 Mill C \& George SJ. Wnt signalling in smooth muscle cells and its role in cardiovascular disorders. Cardiovascular Research 201295 233-240. (doi:10.1093/cvr/cvs141)

9 Tsaousi A, Williams H, Lyon CA, Taylor V, Swain A, Johnson JL \& George SJ. Wnt4/ $\beta$-catenin signalling induces VSMC proliferation and is associated with intimal thickening. Circulation Research $2011 \mathbf{1 0 8}$ 427-436. (doi:10.1161/CIRCRESAHA.110.233999)

10 Christman MA II, Goetz DJ, Dickerson E, McCall KD, Lewis CJ, Benencia F, Silver MJ, Kohn LD \& Malgor R. Wnt5a is expressed in murine and human atherosclerotic lesions. American Journal of Physiology. Heart and Circulatory Physiology 2008294 H2864-H2870. (doi:10.1152/ajpheart.00982.2007)

11 Moester MJC, Papapoulos SE, Lowik CW \& van Bezooijen RL. Sclerostin: current knowledge and future perspectives. Calcified Tissue International 201087 99-107. (doi:10.1007/s00223-010-9372-1)

12 Shao JS, Aly ZA, Lai CF, Cheng SL, Cai J, Huang E, Behrmann A \& Towler DA. Vascular Bmp Msx2 Wnt signaling and oxidative stress in arterial calcification. Annals of the New York Academy of Sciences 2007 1117 40-50. (doi:10.1196/annals.1402.075)

13 Zhu D, Mackenzie NC, Millan JL, Farquharson C \& MacRae VE. The appearance and modulation of osteocyte marker expression during calcification of vascular smooth muscle cells. PLOS ONE 20116 e19595. (doi:10.1371/journal.pone.0019595)

14 Didangelos A, Yin X, Mandal K, Baumert M, Jahangiri M \& Mayr M. Proteomics characterization of extracellular space components in the human aorta. Molecular \& Cellular Proteomics 20109 2048-2062. (doi:10.1074/mcp.M110.001693)

15 Anderson JB, Barnett E \& Nordin BE. The relation between osteoporosis and aortic calcification. British Journal of Radiology 196437 910-912. (doi:10.1259/0007-1285-37-444-910)

16 Gennari L, Merlotti D, Valenti R, Ceccarelli E, Ruvio M, Pietrini MG, Capodarca C, Franci MB, Campagna MS, Calabrò A et al. Circulating sclerostin levels and bone turnover in type 1 and type 2 diabetes. Journal of Clinical Endocrinology and Metabolism 201297 1737-1744. (doi:10.1210/jc.2011-2958)

17 Neumann T, Hofbauer LC, Rauner M, Lodes S, Kästner B, Franke S, Kiehntopf M, Lehmann T, Müller UA, Wolf G et al. Clinical and endocrine correlates of circulating sclerostin levels in patients with type 1 diabetes mellitus. Clinical Endocrinology 201480 649-655. (doi:10.1111/cen.12364)

18 Perk J, DeBacker G, Gohlke H, Graham I, Reiner Z, Verschuren M, Albus C, Benlian P, Boysen G, Cifkova R et al. European Guidelines on cardiovascular disease prevention in clinical practice (version 2012). The Fifth Joint Task Force of the European Society of Cardiology and Other Societies on Cardiovascular Disease Prevention in Clinical Practice (constituted by representatives of nine societies and by invited experts). European Heart Journal 201233 1635-1701. (doi:10.1093/ eurheartj/ehs092)

19 American Diabetes Association. Standards of medical care in diabetes Diabetes Care 201336 S11-S66. (doi:10.2337/dc13-S011)

20 Catalano A, Morabito N, Di Vieste G, Pintaudi B, Cucinotta D, Lasco A $\&$ Di Benedetto A. Phalangeal quantitative ultrasound and metabolic control in pre-menopausal women with type 1 diabetes mellitus. Journal of Endocrinological Investigation 201336 347-351. (doi:10.3275/8646)

21 Glass DA II, Bialek P, Ahn JD, Starbuck M, Patel MS, Clevers H, Taketo MM, Long F, McMahon AP, Lang RA et al. Canonical Wnt signaling in differentiated osteoblasts controls osteoclast differentiation. Developmental Cell 20058 751-764. (doi:10.1016/j.devcel.2005. 02.017) 
22 Lombardi G, Lanteri P, Colombini A, Mariotti M \& Banfi G. Sclerostin concentrations in athletes: role of load and gender. Journal of Biological Regulators and Homeostatic Agents 201226 157-163.

23 Modder UI, Hoey KA, Amin S, McCready LK, Achenbach SJ, Riggs BL, Melton LJ III \& Khosla S. Relation of age, gender, and bone mass to circulating sclerostin levels in women and men. Journal of Bone and Mineral Research 201126 373-379. (doi:10.1002/jbmr.217)

24 Amrein K, Amrein S, Drexler C, Dimai HP, Dobnig H, Pfeifer K, Tomaschitz A, Pieber TR \& Fahrleitner-Pammer A. Sclerostin and its association with physical activity, age, gender, body composition, and bone mineral content in healthy adults. Journal of Clinical Endocrinology and metabolism 201297 148-154. (doi:10.1210/jc.2011-2152)

25 Mirza FS, Padhi ID, Raisz LG \& Lorenzo JA. Serum sclerostin levels negatively correlate with parathyroid hormone levels and free estrogen index in postmenopausal women. Journal of Clinical Endocrinology and Metabolism 201095 1991-1997. (doi:10.1210/jc.2009-2283)

26 García-Fontana B, Morales-Santana S, Varsavsky M, García-Martín A, García-Salcedo JA, Reyes-García R \& Muñoz-Torres M. Sclerostin serum levels in prostate cancer patients and their relationship with sex steroids. Osteoporosis International 201425 645-651.

27 Codner E, Merino PM \& Tena-Sempere M. Female reproduction and type 1 diabetes: from mechanisms to clinical findings. Human Reproduction Update 201218 568-585. (doi:10.1093/humupd/dms024)

28 Tomar R, Dhindsa S, Chaudhuri A, Mohanty P, Garg R \& Dandona P. Contrasting testosterone concentrations in type 1 and type 2 diabetes. Diabetes Care 200629 1120-1122. (doi:10.2337/dc06-0197)

29 van Dam EW, Dekker JM, Lentjes EG, Romijn FP, Smulders YM, Post WJ, Romijn JA \& Krans HM. Steroids in adult men with type 1 diabetes: a tendency to hypogonadism. Diabetes Care 200326 1812-1818. (doi:10.2337/diacare.26.6.1812)

30 Morales-Santana S, García-Fontana B, García-Martín A, RozasMoreno P, García-Salcedo JA, Reyes-García R \& Muñoz-Torres M. Atherosclerotic disease in type 2 diabetes is associated with an increase in sclerostin levels. Diabetes Care 201336 1667-1674. (doi:10.2337/ dc12-1691)

31 Szulc P, Bertholon C, Borel O, Marchand F \& Chapurlat R. Lower fracture risk in older men with higher sclerostin concentration: a prospective analysis from the MINOS study. Journal of Bone and Mineral Research 201328 855-864. (doi:10.1002/ jbmr.1823)
32 Ardawi MS, Rouzi AA, Al-Sibiani SA, Al-Senani NS, Qari MH \& Mousa SA. High serum sclerostin predicts the occurrence of osteoporotic fractures in postmenopausal women: the Center of Excellence for Osteoporosis Research Study. Journal of Bone and Mineral Research 201227 2592-2602. (doi:10.1002/jbmr.1718)

33 Yamamoto M, Yamauchi M \& Sugimoto T. Elevated sclerostin levels are associated with vertebral fractures in patients with type 2 diabetes mellitus. Journal of Clinical Endocrinology and Metabolism 201398 4030-4037. (doi:10.1210/jc.2013-2143)

34 Hampson G, Edwards S, Conroy S, Blake GM, Fogelman I \& Frost ML. The relationship between inhibitors of the Wnt signalling pathway (Dickkopf-1 (DKK1) and sclerostin), bone mineral density, vascular calcification and arterial stiffness in post-menopausal women. Bone 201356 42-47. (doi:10.1016/j.bone.2013.05.010)

35 Claes KJ, Viaene L, Heye S, Meijers B, d'Haese P \& Evenepoel P. Sclerostin: another vascular calcification inhibitor? Journal of Clinical Endocrinology and Metabolism 201398 3221-3228. (doi:10.1210/jc. 2013-1521)

36 Viaene L, Behets GJ, Claes K, Meijers B, Blocki F, Brandenburg V, Evenepoel P \& D'Haese PC. Sclerostin: another bone-related protein related to all-cause mortality in haemodialysis? Nephrology, Dialysis, Transplantation 201328 3024-3030. (doi:10.1093/ndt/gft039)

37 Shao JS, Cai J \& Towler DA. Molecular mechanisms of vascular calcification: lessons learned from the aorta. Arteriosclerosis, Thrombosis, and Vascular Biology 200626 1423-1430. (doi:10.1161/01.ATV. 0000220441.42041.20)

38 Djaberi R, Schuijf JD, Boersma E, Kroft LJ, Pereira AM, Romijn JA, Scholte AJ, Jukema JW \& Bax JJ. Differences in atherosclerotic plaque burden and morphology between type 1 and 2 diabetes as assessed by multislice computed tomography. Diabetes Care 200932 1507-1512. (doi:10.2337/dc09-0320)

39 Funato Y, Michiue T, Asashima M \& Miki H. The thioredoxin-related redox-regulating protein nucleoredoxin inhibits Wnt- $\beta$-catenin signalling through dishevelled. Nature Cell Biology 20068 501-508. (doi:10.1038/ncb1405)

40 Gaudio A, Privitera F, Pulvirenti I, Canzonieri E, Rapisarda R \& Fiore CE. The relationship between inhibitors of the Wnt signalling pathway (sclerostin and Dickkopf-1) and carotid intima-media thickness in postmenopausal women with type 2 diabetes mellitus. Diabetes \& Vascular Disease Research 201411 48-52. (doi:10.1177/ 1479164113510923)

Received 6 February 2014

Revised version received 8 May 2014

Accepted 2 June 2014 